\title{
ADIPOSE DERIVED STEM CELLS- A REVIEW OF THEIR CURRENT ROLE IN RECONSTRUCTIVE SURGERY AND REGENERATIVE MEDICINE
}

\section{Dr. Sheeja Rajan}

\section{Rithwik Rajesh*}

\author{
Professor And Head, Department Of Plastic And Reconstructive Surgery,
} Government Medical College, Kozhikode.

B.Tech (Genetic Engineering), SRM Institute Of Science And Technology, Kattankulathur. * Corresponding Author

ABSTRACT Adipocytes are now known to have immense capacity for self-renewal and as well as multipotential differentiation. Adipose derived stem cells (ADSC) are multipotent stem cells of mesenchymal origin, located ubiquitously in human body. Unlike the bone marrow stem cells, they are easy to harvest from subcutaneous tissues by minimally invasive procedures. Several advances in isolation, invitro culture and replication of adipose derived stem cells have occurred in last decade. Cell assisted lipo-transfers for enhancing the survival of autologous fat grafts (AFG) by enriching them with ADSC is being practiced in Plastic surgery. Because of their potential for angiogenesis, tissue regeneration, immunomodulatory and paracrine effects, fat grafts find considerable applications in Plastic surgery and Regenerative medicine. This paper explores the current research into applications, techniques of harvest, culture and tissue engineering of $\mathrm{ADSC}$ and the concerns about the oncogenic potentiation and genetic stability of the cultured cell lines.

KEYWORDS : Adipose derived stem cells (ADSC), Autologous fat grafts, Gene expressions, Nano-fat, Regenerative medicine, Tissue engineering

\section{INTRODUCTION}

Adipose tissue is no longer considered an energy reservoir, thermal insulator or mechanical buffer of the body alone. Research has shown that Adipocytes have immense capacity for self-renewal and multipotential differentiation into chondrogenic, osteogenic, myogenic and neurogenic cell lineages when exposed to specific inductive environments ${ }^{1}$. Adipose derived stem cells are multipotent stem cells of mesenchymal origin, located ubiquitously in the human body. Unlike the bone marrow stem cells, the ADSC are abundant, self-replenishing and easy to harvest from subcutaneous tissue by minimally invasive procedures ${ }^{2}$. In the past decade, there has been heightened interest into translational research for application of ADSC in regenerative medicine and reconstructive surgery. This paper explores the current research into techniques of harvest, culture and tissue engineering of $\mathrm{ADSC}$, their differentiation into cell lines and the concerns raised into the oncogenic potentiation and genetic stability of the cultured cell lines.

\section{METHOD}

The article was prepared by narrative review of selected articles from Plastic surgical and Biomedical journals, indexed in MEDLINE, PubMed, Research Gate, Scielo and Google scholar using search terms like 'adipose derived stem cells', 'autologous fat grafts', 'fat injections', 'regenerative medicine', 'reconstructive surgery' and 'tissue engineering'. The review included articles in English from the last decade, selected owing to their relevance to the topic or the uniqueness of scientific content.

\section{History of Fat grafting}

Mazzolla et $\mathrm{al}^{3}$ states that the earliest reports in literature on autologous fat grafting was by Neuber in 1893 using small pieces of fat from arm, transferred for contour correction to depressed area of the face resulting from tuberculosis osteitis. Czerny in 1895 used a large lipoma to fill a defect in the breast. However, the early results were not encouraging. Neuhof in 1928 stated that the transplanted autologous fat undergoes same changes as transplanted bone, dies and gets replaced either by fibrous tissue or by newly formed fat after being engulfed by histocyte-like cells. Guerney in 1938 reported similar findings and recommended over-correction of deformities. Fischer and Fischer in 1974 proposed liposuction technique for minimally invasive harvesting of fat grafts.

Fat grafting remained a controversial topic among Plastic surgeons because some surgeons used to get impressive results, but many had disappointing results. The American Society of Plastic and Reconstructive Surgery (ASPRS) Ad-Hoc Committee on New Procedures produced a report on 30 September 1987, long- term, controlled clinical studies are needed before firm conclusions can be made regarding its validity ${ }^{4}$. However, with Coleman's standardised technique for structural fat grafting in 1997, success rates with fat grafts improved dramatically,6. This was attributed to atraumatic technique, lower aspiration pressure, centrifugation to remove excess blood and lysed fat components and injecting fat as tiny aliquot in multiple layers to promote revascularization. Surgical research on further refinements of technique of harvest, separation and additives for fat grafts are continuing.

In 2013, Tonnard et al introduced the "Nano-fat grafting" technique, where mechanically dissociated emulsion of adipose tissue with extracellular matrix, rich in nucleated cells and stem cells was used as injections ${ }^{7}$. Khouri et al stated that only microdroplets with radii less than $1.6 \mathrm{~mm}$ were capable of complete revascularization ${ }^{8}$. Toyserkani et al found that survival rate of the regular lipo-transfer in the human studies varied from 16.3 to $79 \%$. Zhang et al have demonstrated the higher efficiency of heparinized decellularized adipose tissue matrix loaded with b-FGF growth factor in promoting stable neo-adipose tissue formation and neovascularization ${ }^{10}$. Choi J et al reported synergistic effects of Platelet rich plasma (PRP) mixed with ADSCs in improving fat graft survival ${ }^{11}$.

Meanwhile, there were advances in isolation, invitro culture and replication of ADSC. Sattler and Sommer found that frozen and stored fat when thawed contained only fat cells and no fibrous debris ${ }^{12}$. Ullmann et al experimented with multiple serum-free cell culture media (MCDB 153), adding cultured ADSC to aspirated human fat prior to reinjection in mice $^{13}$. Mashiko et al demonstrated the clinical utility of such "cell assisted lipo-transfers" for enhancing the survival of fat grafts ${ }^{14}$. Such a cell-based therapy can be repeated without cell culturing, which used to be a great barrier against active clinical application ${ }^{15}$.

Current state of autologous fat grafting and its clinical applications

Several studies have demonstrated the regenerative potential of autologous fat, presumably because of the ADSC content of the graft. The AFG have shown potential for angiogenesis, nerve regeneration, restoration of dermal thickness and 
scleroderma, radiation therapy, rheumatoid arthritis or osteoarthritis ${ }^{8}$.

\section{Applications in Plastic Surgery}

Autologous fat grafts, either as Structural fat grafts or nano fat grafts are being widely used in several Aesthetic surgical procedures like facial rejuvenation, permanent fillers, scar therapy, eyebrow lift, body contouring and lipoplasty, breast augmentation and genital enhancement. Non-Aesthetic procedures using fat grafts include - skull base surgery, migraine headaches, TMJ ankylosis correction, palatal lengthening, pharyngoplasty, phono-surgery, pectus excavatum, post radiation defects contouring and craniofacial microsomia ${ }^{16}$. They can be used as soft tissue fillers in contour deformities, urethral strictures or stress urinary incontinence. Tamama K and Kerpedjieva SS showed acceleration of all phases of wound healing by modulating multiple growth factors and cytokines including transforming growth factor beta (TGF), granulocyte colony stimulating factor, platelet derived growth factor (PDGF) epidermal growth factor (EGF), tumour necrosis factor, vascular endothelial growth factor, insulin growth factor, keratinocyte growth factor and inflammatory interleukins, secreted from multipotential stromal cells or mesenchymal stem cells ${ }^{17}$.

\section{Applications in Regenerative medicine}

In the presence of specific inducers, ADSC demonstrated the ability to differentiate into multiple mesenchymal cell lines. Better understanding of cellular, molecular, and immunobiological events surrounding cell and tissue transplantation have opened new options for novel therapeutic strategies with $\operatorname{ADSC}^{18,19,20}$. Several cultivation methods, reprogramming, genetic manipulation, epigenetic modulation as well as organisation in three-dimensional matrices by tissue engineering are all showing considerable promise of $\mathrm{ADSC}$ in future cell replacements. In addition, its endocrine activity manifested in the release of adipocytokines, cytokines, transcriptional and growth factors, which forms a secretome has also been subject of considerable research. Adipose tissue is thought to have complex network of interactions with the immune, endocrine, nervous and cardiovascular systems. ADSCs have therefore been used in clinical trials for the treatment of diabetes mellitus, liver disease, corneal lesions, articular and cutaneous lesions ${ }^{21,22}$. Sowa et al have have demonstrated axonal regeneration , formation of myelin and restoration of atrophied muscles with ADSC transplantation ${ }^{23}$. Oishi $\mathrm{K}$ et al recommend cryopreservation and earlier banking of younger adipose tissue-derived cells with a higher regenerative, tissue remodelling, and therapeutic potential to avoid age-related reduction in the regenerative potential of progenitor cells ${ }^{24}$.

Controversy and debate about Adipose-derived stem cell therapy for post-oncologic reconstruction

Though fat grafting has found considerable use in oncoreconstruction, especially after breast cancer surgeries, several researchers have expressed concerns over increased tumour cell viability and reduced apoptotic cell deaths when co cultured with pre-adipocytes ${ }^{25}$. Kamat et al have also expressed concerns over progression and metastatic spread of breast cancer cells in the presence of $\mathrm{ADSC}$, in humanised orthotopic murine models ${ }^{26}$. However, Agha et al performed a meta-analysis with pooled data from 3624 patients have showed that there is no significant oncologic ramifications with fat graft treatments ${ }^{27}$.

Adipose-derived stem cells and Stromal vascular fractionClarifications on terminology and characteristics

According to Gimble et al , there are five criteria for using stem cells for medical purposes: (a) Presence of cells in abundance. (b) Harvesting through minimally invasive procedures. (c)
Regulation of the differentiation of cell lineages. (d) Possibility of their use as autograft. (e) They must be manipulable within the rules of professional practice. ADSCs meet all these criteria $^{28}$. A lipoaspirate contains cells from mesodermal germ layer with a supportive stromal vascular fraction (SVF). SVF consists of a heterogeneous mixture of cells, including endothelial cells, smooth muscle cells, pericytes, leukocytes, mast cells, and pre-adipocytes ${ }^{1,29,30}$. In addition to these cells, the SVF contains an abundant population of multipotent adipose-tissue-derived stem cells (ASCs) that possess the capacity to differentiate into cells of mesodermal origin. The SVF composition was studied using limiting dilution and colony-forming unit (CFU) assays. The capacity of these ASCs to differentiate into the chondrogenic and osteogenic pathways was investigated by quantitative real-time polymerase chain reaction and immuno-histochemistry. The abdomen seems to be preferable to the hip/thigh region for harvesting adipose tissue, yielding SVF cells for stem-cellbased therapies ${ }^{2}$.

In 2006, the International Society for Cellular Therapy (ICTS) defined a set of minimal criteria for identifying cells as ADSCs. These include adherence to tissue culture plastic, maintenance of multipotency with invitro expansions, long term self-renewal capacity, while maintained in standard culture conditions and expression of CD73, CD90 and CD105 while lacking the expression of CD45, CD34, CD14 or CD1 lb, CD79a or CD19 and HLA-DR surface molecules. Along with the International Federation for Adipose Therapeutics and Science in 2013, the ICTS has denoted additional surface markers CD13, CD29 and CD44 as being constitutively expressed at $>80 \%$ on the surface of ADSCs, while CD31, CD45 and CD235a are the primary negative markers that should be expressed on less than $2 \%$ of the cells. The viability of the isolated cells should exceed $70 \%$ and the presence of at least two positive and two negative markers are necessary for foundational phenotyping ${ }^{29}$. Morphologically, ADSC are fibroblast-like cells and preserve their shape after in vitro expansion. Average doubling time of tissue cultured ADSC is between 4 to 5 days. In vitro culturing leads to changes in ADSC immune-phenotype ${ }^{30}$

\section{Isolation and culture of ADSC}

Banyard et al elaborates the technique of ADSC isolation using enzymatic digestion of lipoaspirate with dispase, trypsin or collagenase to release the stromal vascular fraction $(\mathrm{SVF})^{31}$. It is then washed with sterile $1 \%$ PBS until golden in colour, incubated at $37^{\circ} \mathrm{C}$ with intermittent agitation for 30 minutes till cloudy. The infranatant is then carefully aspirated, transferred to $50 \mathrm{ml}$ conical tubes and centrifuged. The supernatant is discarded and resulting pellet, the SVF and resuspended in control media [supplemented with $10 \%$ foetal bovine serum, $500 \mathrm{IU}$ penicillin and $500 \mathrm{lg}$ streptomycin]. The cells are then counted and plated in uncoated T75 flasks. With this technique they report a consistent yield of SVF (>1 $910^{7}$ cells) per $20 \mathrm{mg}$ of lipoaspirate.

\section{Tissue engineering with ADSC}

Adipose tissue engineering is projected as future alternative strategy to abide the plastic surgical principle of "replacing like tissue with like". 3-D biomaterial constructs seeded with ADSC, fibroblasts and additional growth factors in embedded gelatin microspheres, have been shown to support adipogenesis and neovascularization both in-vitro and invivo. Synthetic polymers (poly-lactic acid, poly-glycolic acid or their copolymers, polyethylene terephthalate meshes and silicone tubing) or natural polymers like (collagen, silk fibroin, decellularized human placenta and Matrigel) are being explored for adipose tissue engineering ${ }^{32,33}$.

Gene expressions of Ādipose-derived stem cells Gnanasegaran et al, three groups of mesenchymal stem cells 
were derived from bone marrow (BM-MSC), adipose tissue obtained using liposuction and adipose tissue from biopsy and cultured. BM-MSCs were observed to proliferate better than both groups of ADSCs, with higher cell count, population doubling as well as lower senescence assay ${ }^{34}$. ADSCs from liposuction was better in terms of proliferation rate and had a more homogenous population of stem cells than ADSCs from biopsy ${ }^{35}$.

Only $1 \%$ of ADSCs expressed the HLA-DR molecule (which is an MHC class II cell surface receptor encoded by the human leukocyte antigen complex on chromosome 6 region $6 \mathrm{p} 21.31$.$) ,$ suggesting their prospects for immune modulation and allogeneic transplantation. Since the primary function of HLADR is to present peptide antigens, potentially foreign in origin, to the immune system for the purpose of eliciting or suppressing T-(helper)-cell responses that eventually lead to the production of antibodies against the same peptide antigen $^{36}$.Through pathway analysis, it was discovered that some specific genes like NANOG, CTNNBl, POU5Fl (OCT4) and DNMT3B, which were related to stemness and proliferation were expressed by $\mathrm{ADSCs}{ }^{34}$.

CTNNBl gene plays a major role in WNT signaling in promoting cell proliferation. Also, CTNNBl gene together with WNTl and WNT3A genes inhibit osteogenesis differentiation of $\mathrm{MSCs}^{37}$. RUNX2 was also significantly expressed and was found to suppress osteoblast differentiation by affecting cell cycle progression in the Gl phase ${ }^{38}$. Based on the expression of SOX17 and ISLl, gene expressions in ADSCs (liposuction) were found to be focused towards endoderm lineage. ISLl is an endoderm gene related to beta cell line, and indirectly involved in digestive system formation, while SOX17 plays an important role in the formation of beta cell lines ${ }^{39,40}$. Jun ES et al have produced immortalized ADSC cell lines showing normal karyotypes, enhanced longevity in culture and increased differentiation potential, by stably transducing them with the catalytic subunit of the human telomerase gene (hTERT) ${ }^{41}$. Maitra et al stated that human stem cell lines develop genetic and epigenetic alterations in vitro, implying the need to use them before derivations that could increase their oncogenic potential on repeated cultures ${ }^{42}$.

We conclude that with their multicentric potential for tissue generation, ADSCs have tremendous applications in reconstructive surgery and regenerative medicine.

\section{REFERENCES}

1. Zuk P. Adipose-Derived Stem Cells in Tissue Regeneration: A Review. ISRN Stem Cells. 2013.10.1155/2013/713959.

2. Jurgens WJ, Oedayrajsingh-Varma MJ, Helder MN, Zandiehdoulabi B, Schouten TE, Kuik DJ, Ritt MJ, van Milligen FJ. Effect of tissue-harvesting site on yield of stem cells derived from adipose tissue: implications for cell-based therapies. Cell Tissue Res. 2008;332:415-26.

3. Mazzola RF, Mazzola IC. The fascinating history of fat grafting. J Craniofac Surg. 2013;24:1069-71.

4. Shiffman MA. Autologous Fat Transplantation. The American Journal of Cosmetic Surgery. 1997; 14:433-43.

5. Coleman SR. Facial recontouring with lipostructure. Clin Plast Surg. 1997;24:347-67.

6. Coleman SR. Structural fat grafts: The ideal filler? Clin Plast Surg. 2001;28:111-9.

7. Tonnard P, Verpaele A, Peeters G, Hamdi M, Cornelissen M, Declercq H. Nanofat grafting: basic research and clinical applications. Plast Reconstr Surg. 2013;132:1017-26.

8. Khouri RK Jr, Khouri RK. Current Clinical Applications of Fat Grafting. Plast Reconstr Surg. 2017;140:466e-86e.

9. Toyserkani, Navid \& Quaade, Marlene \& Sørensen, Jens. (2016). Cell-Assisted Lipotransfer: A Systematic Review of Its Efficacy. Aesthetic Plastic Surgery. 40. 10.1007/s00266-016-0613-1.

10. Zhang, Shipin \& Lu, Qiqi \& Cao, Tong \& Toh, Wei Seong. (2016). Adipose Tissue and Extracellular Matrix Development by Injectable Decellularized Adipose Matrix Loaded with Basic Fibroblast Growth Factor. Plastic and reconstructive surgery. 137. 1171-80. 10.1097/PRS.0000000000002019.

11. Choi J, Minn KW, Chang H. The efficacy and safety of platelet-rich plasma and adipose-derived stem cells: an update. Arch Plast Surg 2012; 39: 585-92.

12. Sommer B, Sattler Current concepts of fat graft survival: Histology of aspirated adipose tissue and review of the literature. Dermatol Surg. 2000;26:1159-66.

13. Ullmann Y, Hyams M, Ramon Y, Beach D, Peled IJ, Linderbaum ES. Enhancing the survival of aspirated human fat injected into mice. Plast Reconstr Surg 1998; 101: 1940-44

14. Mashiko $\mathrm{T}$, Yoshimura $\mathrm{K}$. How does fat survive and remodel after grafting? Clin Plastic Surg 2015;42:18le90.

15. Kim YJ, Jeong JH. Clinical application of adipose stem cells in plastic surgery. J Korean Med Sci. 2014 Apr;29:462-7.

16. Tanikawa DY, Aguena M, Bueno DF, Passos-Bueno MR, Alonso N. Fat grafts supplemented with adipose-derived stromal cells in the rehabilitation of patients with craniofacial microsomia. Plast Reconstr Surg. 2013;132:141-52.

17. Tamama $\mathrm{K}$ and Kerpedjieva SS Acceleration of Wound Healing by Multiple Growth Factors and Cytokines Secreted from Multipotential Stromal Cells/Mesenchymal Stem Cells. Adv Wound Care (New Rochelle) 2012; 1 177-82.

18. Tremolada C, Palmieri G, Ricordi C. Adipocyte transplantation and stem cells: plastic surgery meets regenerative medicine. Cell Transplant. 2010;19:1217-23

19. Hristov TG, M. Garcia-Arranz, I. Garcia-Gomez et al. Sutures enriched with adipose-derived stem cells decrease the local acute inflammation after tracheal anastomosis in a murine model. European Journal Cardio-Thoracic Surgery 2012; 42:e40-e47.

20. Wolbank S,G.Stadler, A.Peterbauer etal. Telomerase immortalized human amnion- and adipose-derived mesenchymal stem cells: maintenance of differentiation and immunomodulatory characteristics. Tissue Engineering A 2009; $15: 1843-54$.

21. Miana VV, González EAP. Adipose tissue stem cells in regenerative medicine. Ecancer medical science. 2018;12:822.

22. Si Z, Wang X, Sun C, Kang Y, Xu J, Wang X, Hui Y. Adipose-derived stem cells: Sources, potency, and implications for regenerative therapies. Biomed Pharmacother. 2019;114:108765.

23. Sowa Y, Imura T, Numajiri T, Nishino K, Fushiki S. Adipose-Derived Stem Cells Produce Factors Enhancing Peripheral Nerve Regeneration: Influence of Age and Anatomic Site of Origin. Stem Cells and Development 2012; 21: 1852-62.

24. Oishi K, Noguchi H, Yukawa H, Miyasaki T, Kato R, Kitagawa Y, Ueda M, Hayashi S. Cryopreservation of mouse adipose tissue-derived stem/progenitor cells. Cell Transplant 2008; 17:35-41.

25. Tan SS ,Weiliang Loh. The utility of adipose-derived stem cells and stromal vascular fraction for oncologic soft tissue reconstruction: Is it safe? A matter for debate.The Surgeon 2017; 15: 186-9.

26. Kamat P Riccardo S, Philip K, Souzan S, Maurizio C, Pietro G, Vijay GS Daniel E, Anne-Catherine A, Plock J. Human Adipose-derived mesenchymal stromal cells may promote Breast cancer progression and metastatic spread. Plastic and reconstructive surgery. 2015; 136: 76-84

27. Agha RA, Fowler AJ, Herlin C, Goodacre TE, Orgill DP. Use of autologous fat grafting for breast reconstruction: a systematic review with meta-analysis of oncological outcomes. J Plastic Reconstr Aesthet Surg 2015;68:143e61.

28. Gimble JM, Katz AJ, Bunnell BA. Adipose-derived stem cells for regenerative medicine. Circ Res. 2007;100:1249-60.

29. Oedayrajsingh-Varma MJ, van Ham SM, Knippenberg M, Helder MN, KleinNulend J, Schouten TE, Ritt MJ, van Milligen FJ. Adipose tissue-derived mesenchymal stem cell yield and growth characteristics are affected by the tissue-harvesting procedure. Cytotherapy. 2006;8:166-77.

30. Minteer DM, Marra KG, Rubin JP. Adipose stem cells: biology, safety regulation, and regenerative potential. Clin Plastic Surg 2015;42:169e79.

31. Banyard DA, Ara A. Salibian, Alan D. Widgerow, Gregory R. D. Evans Implications for human adipose-derived stem cells in plastic surgery J. Cell. Mol. Med. 2015;19:21-30

32. Casadei A, Epis R, Ferroni L, Tocco I, Gardin C, Bressan E, Sivolella S, Vindigni V, Pinton P, Mucci G, Zavan B. Adipose tissue regeneration: a state of the art. J Biomed Biotechnol. 2012;2012:462543.

33. Martinello T, Bronzini I, Volpin A, Vindigni V, Maccatrozzo L, Caporale G, Bassetto F. Patruno M. Successful recellularization of human tendon scaffolds using adipose-derived mesenchymal stem cells and collagen gel. J Tissue Eng Regen Med. $2014 ; 8: 612-9$.

34. Gnanasegaran N, Govindasamy V, Musa S, Kasim NH. Different isolation methods alter the gene expression profiling of adipose derived stem cells. Int $J$ Med Sci. 2014:11:391-403.

35. Scherberich A, Maggio NDD, Mc Nagny KM. A familiar stranger: CD34 expression and putative functions in SVF cells of adipose tissue. World J Stem Cells. 2013;5:1-8.

36. Schreml S, Babilas P, Fruth S, Orsó E, Schmitz G, Mueller MB, Nerlich M, Prant L. Harvesting human adipose tissue-derived adult stem cells: resection versus liposuction. Cytotherapy. 2009; 11:947-57.

37. Boland GM, Perkins G, Hall DJ, Tuan RS. Wnt 3a promotes proliferation and suppresses osteogenic differentiation of adult human mesenchymal stem cells. J Cell Biochem. 2004;93:1210-30.

38. Lucero CM, Vega OA, Osorio MM, Tapia JC, Antonelli M, Stein GS, van Wijnen AJ. Galindo MA. The cancer-related transcription factor Runx2 modulates cell proliferation in human osteosarcoma cell lines. J Cell Physiol. 2013;228:714-23.

39. Guo T, Wang W, Zhang H, Liu Y, Chen P, Ma K, Zhou C. ISLl Promotes Pancreatic Islet Cell Proliferation. PLoS ONE 201 1;6:e22387.

40. Chandra VGS, Muthyala S, Jaiswal AK, Bellare JR, Nair PD, Bhonde RR. IsletLike Cell Aggregates Generated from Human Adipose Tissue Derived Stem Cells Ameliorate Experimental Diabetes in Mice. PLoS ONE. 201 1;6:e20615.

41. Jun ES, T. H. Lee, H. H. Cho, S. Y. Suh, J. S. Jung. Expression of telomerase extends longevity and enhances differentiation in human adipose tissuederived stromal cells. Cellular Physiology and Biochemistry 2004; 14: 261-68.

42. Maitra A, Arking DE, and Shivapurkar N, et al Genomic alterations in cultured human embryonic stem cells. Nature Genetics 2005; 37:1099-1 103 